Relations industrielles

Industrial Relations

\title{
Human Relations in Administration: par Robert Dubin, Prentice-Hall, Inc. Englewood Cliffs, N. J. 2e édit. 1961, 635 pp.
}

Jean-Réal Cardin

Volume 17, numéro 2, avril 1962

URI : https://id.erudit.org/iderudit/1021642ar

DOI : https://doi.org/10.7202/1021642ar

Aller au sommaire du numéro

Éditeur(s)

Département des relations industrielles de l’Université Laval

ISSN

0034-379X (imprimé)

1703-8138 (numérique)

Découvrir la revue

Citer ce compte rendu

Cardin, J.-R. (1962). Compte rendu de [Human Relations in Administration: par Robert Dubin, Prentice-Hall, Inc. Englewood Cliffs, N. J. 2e édit. 1961, 635 pp.] Relations industrielles / Industrial Relations, 17(2), 212-213.

https://doi.org/10.7202/1021642ar

Tous droits réservés (C Département des relations industrielles de l’Université Laval, 1962
Ce document est protégé par la loi sur le droit d'auteur. L'utilisation des services d'Érudit (y compris la reproduction) est assujettie à sa politique d'utilisation que vous pouvez consulter en ligne.

https://apropos.erudit.org/fr/usagers/politique-dutilisation/ 


\section{RECENSIONS}

Human Relations in Administration: par Robert Dubin, Prentice-Hall, Inc. Englewood Cliffs, N. J. 2e édit. 1961, $635 \mathrm{pp}$.

- Il s'agit ici de la deuxième édition de l'ouvrage du sociologue américain Robert Dubin, ouvrage paru pour la première fois en 1951. Dubin est un des noms les plus connus dans les cercles de la sociologie du travail aux EtatsUnis. En plus de nombreux articles et ouvrages en collaboration consacrés à différents aspects de relations industrielles, Dubin est l'auteur d'au moins deux traités de sociologie du travail publiés par la maison d'édition Prentice-Hall dans la série \&Industrial Relations and Personnel », dont fait partie le présent volume. Les deux traités sont \& The World of Work 》 et \&Working UnionManagement Relations 》 publiés tous deux en 1958.

c Human Relations in Administration », tout en étant d'abord un traité systématique sur les problèmes humains reliés à l'administration de l'industrie contemporaine, se présente avant tout comme un répertoire sélectif et ordonné de textes d'auteurs différents correspondant dans leur présentation aux diverses phases généralement reconnues par la théorie en administration, et dont Dubin est en quelque sorte à la fois l'éditeur et le commentateur.

Il est bon de noter que la présente édition contient plus de vingt-cinq nouveaux textes ajoutés à ceux de la première édition. Enfin, une partie considérable de l'ouvrage est consacrée à l'exposé de «cas 》 réels, quoi qu'amputés d'une partie de leur réalité pour fins de publication et d'étude, sur des problèmes de relations humaines en administration. De larges marges sont ménagées à côté du texte afin de permettre à l'usager du volume de noter ses remarques au fur et à mesure de la lecture qu'il en fait.

Il est aussi nécessaire de reconnaître que le présent ouvrage ne traite pas de l'administration des affaires en général; ce n'est pas un traité sur la théorie de l'administration considérée sous tous ses aspects. Ce qui fait l'objet exclusif du livre de Dubin, c'est essentiellement le facteur humain des organisations industrielles, ainsi que les relations et les problèmes auxquels donne lieu leur administration. Sont donc délaissés les autres «plans » de l'Administration, c'està-dire ceux ayant trait aux facteurs «non-humains » de l'organisaiton: les matières premières, les machines et le capital argent.

Tout au cours du volume, les différents auteurs s'astreignent chacun dans son sujet, à éclairer la dimension hımaine et sociale du fonctionnement administratif des entreprises industrielles. C'est un truisme que d'affirmer que l'entreprise moderne est devenue quelque chose de complexe et d'extrêmement ra. mifiée. Mais une fois cette assertion énoncée, c'est une autre affaire que d'en entreprendre l'étude, d'en saisir les rouages essentiels, d'en caractériser les principales phases et d'en analyser les comportements et les problèmes qui en découlent.

Pourtant c'est à cela qu'il faut arriver, si l'on veut comprendre le monde dans lequel vivent et travaillent des nombres si considérables d'humains dans les sociétés industrialisées. Une semblable connaissance est avant tout requise de ceux qui ont charge de diriger les opérations de nos entreprises industrielles. Le dirigeant d'entreprises présentement ne peut plus considérer son rôle comme il pouvait le faire au début de la Révolution industrielle. L'autorité, sa délégation ainsi que le contrôle des ugisiements des subordonnés ne sont plus les seules exigences du directeur d'ontreprises. S'il veut être efficace non seulement «techniquement», mais aussi «humainement» il doit se conrevoir non plus comme un simple administrateur de «choses » mais aussi et surtout comme un dirigeant d'hommes. Pour y arriver, une connaissance profonde des implications humaines de l'administation lui est nécessaire. C'est de cette connaissance que les textes présentés par Dubin traitent sous ses différents aspects. 
Le livre se divise en sept parties, elles-mêmes subdivisées en différents sujets à l'intérieur desquels prennent place les textes et les remarques personnelles de l'auteur. On y aborde d'abord certains problèmes reliés à la nature même de l'entreprise et aux éléments qui la composent. La deuxième partie traite des organisations industrielles et en fait en quelque sorte la sociologie. Les parties trois, quatre et cinq forment un tryptique consacré: a) au personnel adiministratif, ses différents types, ses problèmes et son organisation; b) aux relations dans l'administration, envisagées selon leurs éléments de pouvoir, d'autorité et de status; c) à l'action administrative où l'on discute des comrnunications, de la prise des décisions, du leadership, de la subordination et lu contrôle. La sixième partie s'intéresse plus précisément aux contextes internes et externes conditionnant la vie de l'entreprise sur les plans technologique et sociétaire. Enfin la dernière partie contient l'ensemble des «cas» présentés.

Encore une fois, ce ne sont pas tant ici les thèmes présentés qui offrent du nouveau, que l'intégration systé natiq' ’e qui en est faite par l'auteur et qui, à notre sens, fournit au lecteur in compendium des plus utiles sur un suiet par ailleurs assez éparpillé, et dans son traitement et dans la conception que souvent l'on s'en fait.

\section{Jean-Réal Cardin}

\section{THE MACHINISTS; A new Study in} American Trade Unionism; par Mark Perman, Wertheim Publications in Industrial Relations, Harvrad University Press, Cambridge, Mass., 1961, 333 pp. Publié au Canada par: S. J. Reginald Saunders and Company Limited, Toronto.

- On a eu trop tendance, depuis quelques années en relations industrielles, à négliger la perspective historique. Depuis l'oeuvre monumentale de Commons et de ses associés sur l'histoire du mouvement syndical aux Etats-Unis, plusieurs études historiques, et d'excellentes, ont sans doute pris place dans la littérature du travail, mais il reste que, bien qu'incluse dans les curriculums universitaires, l'étude de l'histoire des relations du travail ne fait pas en général l'objet de cours y étant spécifiquement consacrés. On étudie l'histoire à l'occasion des études analytiques sur les relations industrielles, les systèmes comparés, les structures, le gouvernement et l'action des syndicats.

La raison en est, à notre avis, que beaucoup de traités historiques ne se sont attachés qu'à la chronologie des faits sans assez utiliser ce fichier des événemnets dans le temps comme facteur d'explication des attitudes, des structures et des problèmes actuels des institutions du travail dont la connaissance est essentielle à une compréhension objective des relations du travail.

Evidemment, il s'agit encore ici d'un ouvrage américain. Sur le plan histcrique, la littérature canadienne est des plus pauvres, et nous attendons toujours l'avènement d'une oeuvre définitive et complète devant servir de point de départ à l'éclosion de toute une série d'oeuvres complémentaires sur le plan :nonographique et spécialisé. Depuis les travaux de Margaret MacKintoch et l'ouvrage de Logan, excepté peut-être l'étude de Trudeau et de ses collaborateurs sur la grève de l'amiante, et certaines recherches plus réduites poursuivies en vue de l'obtention de degrés iniversitaires, l'ensemble des écrits touchant à l'histoire en relations de travail na pas fait, en général, état de recherclıes originales et n'a traité des faits historiques que d'une façon incidente, supesticielle, chématisée à l'extrême, à partir des travaux, pourtant plus qu'incomplets, des pionniers et des quelques témcins de notre histoire sociale qui ont bien voulu nous léguer quelques éléments testamentaires de leur propre action.

Le présent ouvrage fait partie r'un vaste projet de recherche sur l'histoire des relations ouvrières-patronales aux Hats-Unis enstrepris par I'Université Harvard en 1954, et basé sur la reconnaissance de l'urgence d'une revalorisation de l'histoire au sein de l'étude de relations industrielles.

L'association internationale des Machinistes est un des syndicats nord-américains les plus importants à la fois quant au nombre de ses adhérents (près d'un million de membres) et quant à la philosophie qu'il représente dans l'ensemble du mouvement syndical, aussi bien aux Etats-Unis qu'au Canada.

Fondé en 1888 sur la base du métier pur parmi les ouvriers spécialisés en mé- 